\title{
Avaliação dos serviços de assistência ocular em população urbana de baixa renda da cidade de São Paulo - Brasil
}

\author{
Eye care services evaluation in a low-income urban population \\ of São Paulo City - Brazil
}

\author{
Josenilson Martins Pereira ${ }^{1}$ \\ Solang'e Rios Salomão ${ }^{2}$ \\ Rafael Werneck Cinoto ${ }^{3}$ \\ Luana Mendieta ${ }^{4}$ \\ Paula Yuri Sacai ${ }^{5}$ \\ Adriana Berezovsky ${ }^{6}$ \\ Rubens Belfort Júnior ${ }^{7}$
}

Trabalho realizado no Departamento de Oftalmologia da Universidade Federal de São Paulo - UNIFESP - São Paulo (SP) - Brasil.

Mestre em Ciências Visuais e Tecnólogo Oftálmico do Setor de Eletrofisiologia Visual Clínica do Departamento de Oftalmologia da Universidade Federal de São Paulo - UNIFESP - São Paulo (SP) - Brasil.

${ }^{2}$ Professora Associada Livre Docente do Departamento de Oftalmologia da UNIFESP - São Paulo (SP) - Brasil. ${ }^{3}$ Psicólogo e pós-graduando em Ciências Visuais pela UNIFESP - São Paulo (SP) - Brasil.

${ }^{4}$ Mestre em Ciências Visuais pela UNIFESP - São Paulo (SP) - Brasil.

${ }^{5}$ Pós-graduanda em Ciências Visuais e Tecnóloga oftálmica do Setor de Eletrofisiologia Visual Clínica do Departamento de Oftalmologia da UNIFESP - São Paulo (SP) - Brasil

${ }^{6}$ Doutora em Ciências e Professora Adjunta do Departamento de Oftalmologia da UNIFESP - São Paulo (SP) Brasil.

Professor Titular do Departamento de Oftalmologia da UNIFESP - São Paulo (SP) - Brasil.

Endereço para correspondência: Josenilson Martins Pereira. Departamento de Oftalmologia - UNIFESP. Rua Botucatu, 822 - São Paulo (SP) CEP 04023-062 E-mail: jpereiraarthur@yahoo.com.br

Recebido para publicação em 25.07.2007

Última versão recebida em 05.02.2009

Aprovação em 20.05.2009

Apoio: Organização Mundial da Saúde (Contrato TSA PBD.3/15 com o Instituto da Visão da UNIFESP, sob os auspícios do National Eye Institute do National Institutes of Health [Bethesda, Maryland] contrato $n^{\circ}$ N01-EY-2103); Fundação de Amparo à Pesquisa do Estado de São Paulo (FAPESP), auxílio regular à pesquisa $\mathrm{n}^{\circ}$ 04/06670/-9 para SRS, bolsa de doutorado direto $n^{\circ}$ 06/01354-7 para RWC; Fundo de Apoio aos Docentes e Alunos da UNIFESP (FADA); bolsistas de produtividade em pesquisa AB, RBJ e SRS; Conselho Nacional de Desenvolvimento Científico e Tecnológico, bolsistas de produtividade em pesquisa $\mathrm{AB}, \mathrm{RBJ}$ e SRS.

Nota Editorial: Depois de concluída a análise do artigo sob sigilo editorial e com a anuência da Dra. Norma Helen Medina sobre a divulgação de seu nome como revisora, agradecemos sua participação neste processo.

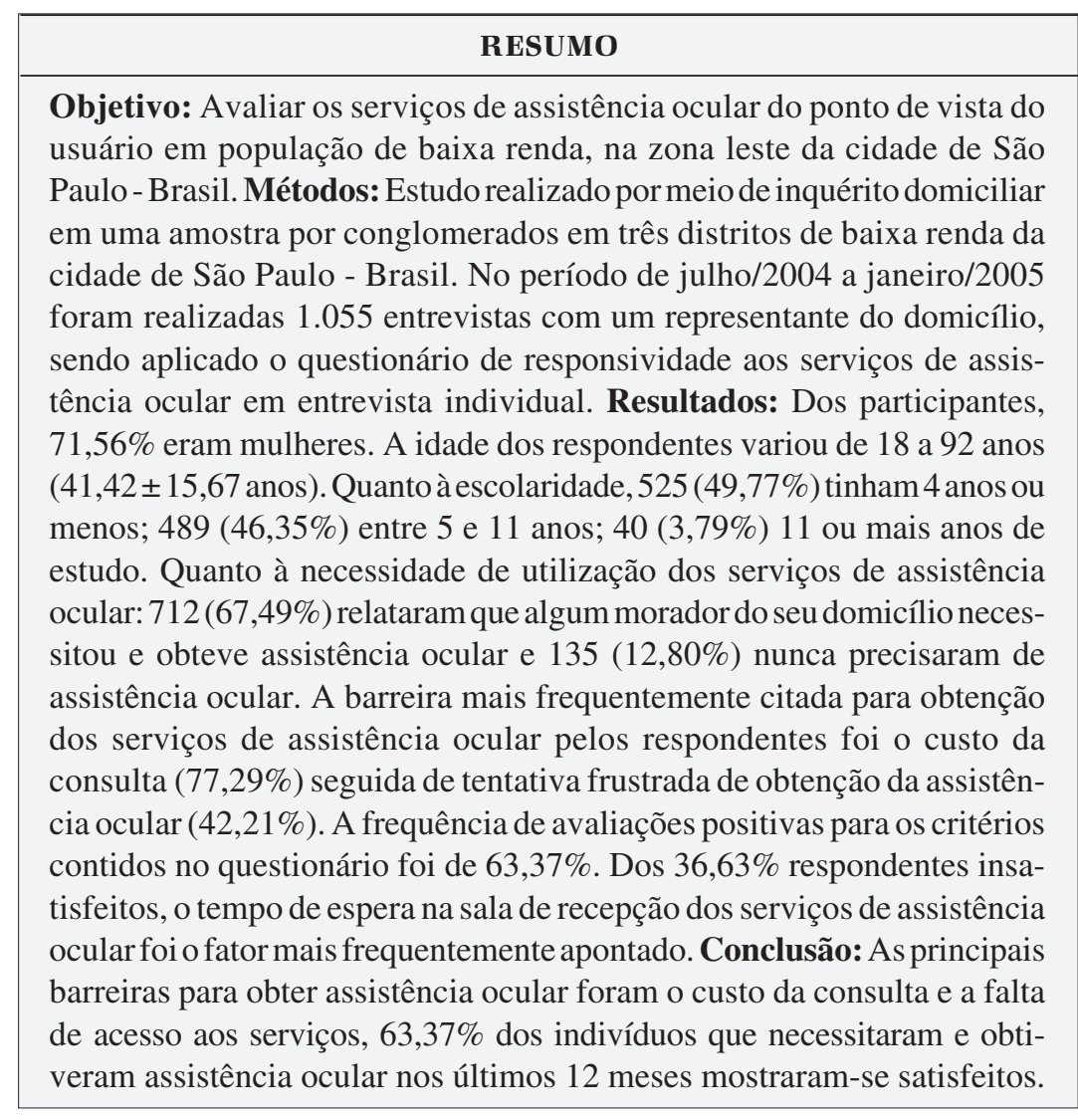

Descritores: Satisfação do paciente; Serviços de saúde ocular; Qualidade da assistência à saúde; Garantia da qualidade dos cuidados de saúde; Oftalmopatias/epidemiologia; Serviços urbanos de saúde

\section{INTRODUÇÃO}

A avaliação do desempenho dos sistemas de saúde tem importância crescente $^{(1)}$. A Organização Mundial da Saúde (OMS) propôs uma metodologia para avaliação do desempenho dos sistemas de saúde em que um dos aspectos abordados é a resposta do usuário ao sistema de saúde (responsividade). Na avaliação dos serviços de saúde são considerados aspectos não clínicos de como o indivíduo é tratado e o ambiente em que seu tratamento é feito ${ }^{(2)}$. 
Na revisão da literatura e em encontro de especialistas em satisfação da população e em qualidade, realizados em 1999, foram identificados oito aspectos para a análise da satisfação do ponto de vista do usuário de qualquer sistema de saúde: dignidade, autonomia, privacidade, comunicação, prontidão no atendimento, acesso a serviços de apoio social durante o tratamento, infraestrutura básica de qualidade e escolha ${ }^{(3)}$.

Com relação à saúde ocular, o aumento progressivo da cegueira e da deficiência visual no mundo pode ser atribuído, em grande parte, à escassez de serviços especializados, às dificuldades de acesso da população à assistência oftalmológica, às dificuldades econômicas, à ausência/insuficiência de esforços educativos que desenvolvam a adoção de comportamento preventivo, e ao envelhecimento populacional ${ }^{(4)}$.

Devido à situação atual e à incorporação de novas tecnologias neste campo tornou-se necessária uma avaliação periódica dos serviços de saúde, especialmente uma avaliação da perspectiva do usuário, que é uma iniciativa fundamental na melhoria da prática profissional e da organização dos serviços de saúde ${ }^{(5-7)}$. Esses dados são relevantes na elaboração de políticas governamentais de saúde ${ }^{(8)}$.

O objetivo deste estudo é avaliar os serviços de assistência ocular do ponto de vista do usuário em população de baixa renda da zona leste da cidade de São Paulo, abordando a necessidade e obtenção, as barreiras e a experiência com a assistência ocular.

\section{MÉTODOS}

Esta pesquisa foi aprovada pelo Comitê de Ética em Pesquisa da Universidade Federal de São Paulo/EPM e pelo Comitê Secretário de Pesquisa Envolvendo Seres Humanos da Organização Mundial de Saúde.

A população do estudo foi recrutada em três distritos de baixa renda da zona leste da cidade de São Paulo: Vila Jacuí, São Miguel e Ermelino Matarazzo. O estudo realizado foi observacional transversal e realizado por meio de inquérito domiciliar em uma amostra por conglomerados. O instrumento utilizado no inquérito domiciliar constava de questionário estruturado sobre a avaliação da assistência ocular - Questionário de Responsividade ao Sistema de Assistência Ocular (QRSAO) e de formulário de enumeração utilizado para obter os dados demográficos de todos os residentes no domicílio.

O QRSAO (Anexo) é um instrumento específico que contém 46 questões (34 fechadas e 12 abertas) abordando diversos aspectos sobre a assistência ocular e foi desenvolvido pela OMS a partir do questionário da Pesquisa Mundial de Saúde ${ }^{(1)}$. O questionário foi traduzido para o português-brasileiro, adaptado para a realidade cultural e econômica nacional, retraduzido para o inglês americano por profissional especializado e comparado à versão original em inglês com grande similaridade. O QRSAO é um instrumento inédito, que foi utilizado pela primeira vez neste estudo, e aborda dois tópicos principais: a necessidade mais recente de assistência ocular dentro do domicílio e uma avaliação, baseada no usuário, sobre a experiência com a assistência ocular no último ano pela experiência relatada pelo respondente para seu próprio atendimento ou para o atendimento de uma criança dependente acompanhada. As questões inferiam sobre prontidão no atendimento, dignidade, comunicação, autonomia, privacidade, escolha e infraestrutura.

Os critérios de inclusão do estudo foram: concessão de termo de consentimento livre e esclarecido, questionários obtidos por entrevista no próprio domicílio do respondente, preenchimento completo e correto do formulário de enumeração e respondente com idade igual ou maior a 18 anos.

O mapeamento da área geográfica do estudo e de todos os domicílios dentro de cada conglomerado foi realizado pela equipe de enumeração e entrevista para delimitar o perímetro e os domicílios que poderiam fazer parte do estudo.

Os dados das entrevistas foram incluídos com auxílio do aplicativo Epi Info 2002 em formulários especificamente desenvolvidos para este estudo que permitiram a verificação de consistência interna dos dados. O aplicativo Stata (versão 8.0) foi utilizado para análise descritiva dos dados.

\section{RESULTADOS}

A amostra analisada foi de 1.055 questionários obtidos a partir de um total de 1.597 domicílios mapeados. A tabela 1 mostra a distribuição de sexo, idade, escolaridade e renda domiciliar dos respondentes. A maioria dos participantes foi do sexo feminino $(71,56 \%)$. A idade dos respondentes variou de 18 a 92 anos (média $=41,42$ e desvio padrão $= \pm 15,67$ anos). Destes 1.055 respondentes, $525(49,77 \%)$ tinham quatro anos ou menos de estudo, 489 (46,35\%) tinham entre cinco e onze anos de estudo, 40 (3,79\%) tinham mais de onze anos de estudo e $1(0,09 \%)$ não respondeu a esta questão. No grupo estudado $49,00 \%$ tinha renda domiciliar menor ou igual a três salários mínimos.

A tabela 2 mostra os resultados obtidos para a questão sobre a necessidade e obtenção de assistência ocular e se esta necessidade foi ou não atendida. Dos 1.055 respondentes, $712(67,49 \%)$ relataram que ele(a) próprio(a) ou algum morador do seu domicílio necessitaram e obtiveram assistência ocular. Quanto ao restante da amostra, $199(18,86 \%)$ precisaram e não obtiveram assistência ocular e $135(12,80 \%)$ nunca precisaram de assistência ocular. No total, 911 respondentes relataram necessidade de assistência ocular $(86,35 \%)$.

Dentre os 712 respondentes que relataram necessidade e obtenção de assistência ocular, 218 (30,62\%) responderam que receberam assistência ocular no último ano para si próprio e $55(7,72 \%)$ responderam que acompanharam uma criança que recebeu assistência ocular. Em suma, 273 (25,88\%) dos 1.055 respondentes utilizaram serviços de assistência ocular no último ano. 
Endereço do domicílio:

Nome do Respondente:

\section{Necessidade de Assistência ocular e Avaliação Geral dos Sistemas de Assistência ocula}

Q7000 de assistência ocular mas não foi possível obtê-la?

[Entrevistador: pare de ler assim que o respondente tiver selecionado uma resposta.]

Q7001

Esta última vez que houve necessidade e não foi conseguida assistência ocular foi para você, para uma criança na sua família, ou para algum outro membro adulto da sua família? [Entrevistador: Use "você" ou "essa criança" ou "outro adulto de seu domicílio" de acordo com a resposta]

Q7002

Que problema no olho, melhor descreve por que você [a criança ou o outro membro de seu domicílio] precisou de assistência ocular?

[Entrevistador - o respondente deve selecionar SOMENTE uma resposta]
1. Nos últimos 30 dias

2. Entre 1 mês e menos de 6 meses atrás

3. Entre 6 meses e menos de 1 ano atrás

4. Entre 1 ano e menos de 2 anos atrás

5. Há mais de dois anos atrás

6. Precisou e obteve assistência ocular

7. Nunca precisou

8. Não sabe

1. Você

2. Uma criança do seu domicílio

3. Outro membro adulto de seu domicílio

(Especifique)

1. Visão embaçada, borrada ou reduzida

2. Problema com luz, ofuscamento ou halos

3. Olho vermelho

4. Dor, incômodo ou coceira no olho

5. Olho lacrimejando ou secreção

6. Ferimento no olho

7. Outro (Especifique)

Que motivos explicam melhor por que você [essa criança ou o outro membro de seu domicílio] não conseguiu assistência ocular?

Q7004

Q7005

Q7006

Q7007

Q7008

Q7009

Q7010

Q7011

Q7012

Q7013

Q7014

Q7015

Q7020

Em geral você diria que está muito satisfeito, parcialmente satisfeito, nem satisfeito nem insatisfeito parcialmente insatisfeito ou muito insatisfeito com as condições dos serviços de assistência ocular na sua região do país.

Se 6 vá para $\mathbf{Q 7 0 2 0}$

Se 7 ou 8 , termine a entrevista

$+2$ 


\section{Consultando Prestadores de Assistência Ocular}

Q7200 No último ano, você recebeu alguma assistência ocular?

Q7201 No último ano, alguma vez você acompanhou uma criança do seu domicílio para cuidar da visão?

1. Sim Não

Se Sim, vá para Q7300

Experiência sobre a Assistência Ocular

[Entrevistador: use "você" ou "esta criança acompanhada" de acordo com as respostas da sessão anterior "Consultando Prestadores de Assistência Ocular"]

Q7300 Qual o nome do último prestador de assistência ocular que você [esta criança acompanhada] consultou? [Entrevistador: tente obter o nome da clínica ou centro de saúde, além do médico, se o respondente foi a uma clínica ou centro de saúde.]

Q7301 O último local em que você [esta criança acompanhada] se consultou era:

Que tipo de prestador de assistência ocular você [esta criança acompanhada] utilizou na última vez?

[Entrevistador: Depois da Q7302 substitua o tipo de prestador de assistência ocular selecionado pelo paciente quando você vir [prestador de assistência ocular] entre parênteses.]

Na sua opinião, as habilidades do [prestador de assistência ocular] foram adequadas para o seu [esta criança acompanhada] tratamento?

Q7303a Como você pagou pelo serviço?

Q7304 Na sua opinião, o material médico e equipamentos do [prestador de assistência ocular] foram adequados para seu [esta criança acompanhada] tratamento?

Q7305 Pensando sobre sua [esta criança acompanhada] última consulta, quanto tempo você demorou para chegar ao local?

Q7306 Pensando sobre sua [esta criança acompanhada] última consulta, como você chegou lá? [Entrevistador: marque aquele usado para a maior parte do percurso]

Q7307 O valor da consulta [do prestador de assistência ocular] incluindo freqüências clínicas e do centro de saúde

Q7308 Material médico, incluindo óculos

Q7309 Exames

Q7310 Transporte

Q7311 Outros (Especifique)
(Quantia)

(Quantia)

(Quantia)

(Quantia)

(Quantia)
Q7312 Para sua [esta criança acompanhada] última consulta, como você classificaria o tempo de viagem até o [prestador de assistência ocular]?
(Especifique)

1. Hospital/clínica pública

2. Hospital/clínica particular

3. Hospital/clínica do convênio

4. Outros (Especifique)

8. Não sabe

1. Médico clínico geral

2. Oftalmologista

3. Optometrista

4. Técnologo oftálmico/paramédico

5. Óptico

6. Curandeiro

7. Outros (Especifique)

8. Não sabe

1. Sim

5. Não

8. Não sabe

1. Particular, sem reembolso

3. Não pagou/sistema público

1. Sim 5. Não

2. Plano de saúde

Horas:

1. Veículo particular 2. Táxi 3. Transporte público

4. Ambulância

\section{Minutos:}

5. Bicicleta

6. Andou

8. Não sabe

7. Outro
8. Não sabe

8. Não sabe 9. Não aplicável, não teve

8. Não sabe $\quad$ 9. Não aplicável, não teve

8. Não sabe 9. Não aplicável, não teve

8. Não sabe

9. Não aplicável, não teve
1. Ótimo
2. Bom
3. Regular

4. Ruim

5. Péssimo

9. Não aplicável 
Q7313 Para sua [esta criança acompanhada] última consulta, como você classificaria o tempo que você esperou antes de ser atendido?

Q7314 Para sua [esta criança acompanhada] última consulta, como você classificaria sua experiência de ter sido recebido e tratado com respeito?

Q7315 Para sua [esta criança acompanhada] última consulta, como você classificaria como sua privacidade foi respeitada durante exames e tratamentos?

Q7316 Para sua [esta criança acompanhada] última consulta, como você classificaria a clareza com que o [prestador de assistência ocular] explicou as coisas para você?

Q7317 Para sua [esta criança acompanhada] última consulta, como você classificaria a experiência de ter tempo suficiente para fazer perguntas sobre seu problema na visão ou tratamento? Para sua [esta criança acompanhada] última consulta, como você classificaria a experiência de ter informações sobre outros tipos de exames ou tratamentos?

Q7319 Para sua [esta criança acompanhada] última consulta, como você classificaria a experiência de estar envolvido em tomar decisões sobre sua assistência ocular ou tratamento?

Para sua [esta criança acompanhada] última consulta, como você classificaria a maneira que o serviço de saúde assegurou que você poderia falar reservadamente com o [prestador de assistência ocular]?

Q7321 Para sua [esta criança acompanhada] última consulta, como você classificaria a liberdade que teve para escolher o seu [prestador de assistência ocular]?

Para sua [esta criança acompanhada] última consulta, como você classificaria a limpeza das salas dentro do local de exame, incluindo os banheiros?

Q7323 Para sua [esta criança acompanhada] última consulta, como você classificaria a quantidade de espaço na sala de espera e na sala onde foi examinado?

\section{Vinhetas de Assistência Ocular}

As perguntas que farei a seguir são sobre experiências hipotéticas de assistência ocular.

Você deverá classificar a experiência na mesma escala que você usou para classificar a sua própria experiência.

Q7401 Mário foi informado que tinha glaucoma e que ele precisava tomar um remédio. O médico rapidamente explicou a condição. O médico está muito ocupado e há uma fila de pacientes esperando por ele. Mário gostaria de saber mais sobre o que ele tem, mas sente que não há tempo para fazer perguntas. O médico diz até logo para Mário, e Mário deixa a clínica.

Patrícia foi a uma clínica cheia. Primeiramente, ninguém a recebeu, mas depois de esperar por 5 minutos uma enfermeira a chamou para a área de exames onde ela foi examinada atrás de uma pequena divisão que a escondia quase totalmente dos outros pacientes.

Roberto teve um ferimento no olho. O médico explicou diferentes maneiras de tratá-lo e a segui pediu alguns exames de sangue. Roberto não sabia por que precisava de exames de sangue e ficou preocupado até que o médico explicou para que seriam os exames.

\begin{tabular}{|c|c|c|}
\hline $\begin{array}{l}\text { 1. Ótimo } \\
\text { 4. Ruim }\end{array}$ & $\begin{array}{l}\text { 2. Bom } \\
\text { 5. Péssimo }\end{array}$ & 3. Regular \\
\hline $\begin{array}{l}\text { 1. Ótimo } \\
\text { 4. Ruim }\end{array}$ & $\begin{array}{l}\text { 2. Bom } \\
\text { 5. Péssimo }\end{array}$ & 3. Regular \\
\hline $\begin{array}{l}\text { 1. Ótimo } \\
\text { 4. Ruim }\end{array}$ & $\begin{array}{l}\text { 2. Bom } \\
\text { 5. Péssimo }\end{array}$ & 3. Regular \\
\hline
\end{tabular}


Dos $199(18,86 \%)$ casos em que houve necessidade e não se obteve assistência ocular, $112(56,28 \%)$ foram para o próprio respondente, $28(14,07 \%)$ foram para uma criança residente no domicílio e $59(29,65 \%)$ para outro adulto residente no domicílio. A maioria, 136 (68,34\%), dos respondentes que não conseguiram assistência ocular relatou que o motivo mais frequente para procurar assistência foi visão embaçada, borrada ou reduzida, seguido de dor, incômodo ou coceira no olho, 23 casos $(11,53 \%)$.

\begin{tabular}{|c|c|c|}
\hline & Frequência & Porcentagem \\
\hline \multicolumn{3}{|l|}{ Idade (anos) } \\
\hline$\leq 20$ & 73 & 6,92 \\
\hline $21-30$ & 222 & 21,04 \\
\hline $31-40$ & 252 & 23,89 \\
\hline $41-50$ & 234 & 22,18 \\
\hline $51-60$ & 128 & 12,13 \\
\hline$>60$ & 146 & 13,84 \\
\hline \multicolumn{3}{|l|}{ Sexo } \\
\hline Masculino & 300 & 28,44 \\
\hline Feminino & 755 & 71,56 \\
\hline \multicolumn{3}{|l|}{ Escolaridade } \\
\hline Nenhuma & 79 & 7,49 \\
\hline$<$ Primário & 117 & 11,09 \\
\hline Primário (até $4^{\underline{a}}$ série) & 329 & 31,19 \\
\hline Fundamental ( $5^{\underline{a}}$ a $8^{\underline{a}}$ série) & 211 & 20,00 \\
\hline Ensino médio & 278 & 26,35 \\
\hline Superior ou maior & 40 & 3,79 \\
\hline Não respondeu & 1 & 0,09 \\
\hline \multicolumn{3}{|l|}{ Renda domiciliar* } \\
\hline Nenhuma & 53 & 5,02 \\
\hline $0-1 \mathrm{SM}$ & 136 & 12,89 \\
\hline $1-3 \mathrm{SM}$ & 328 & 31,09 \\
\hline $3-5 \mathrm{SM}$ & 152 & 14,41 \\
\hline $5-10 \mathrm{SM}$ & 87 & 8,25 \\
\hline$>10 \mathrm{SM}$ & 18 & 1,71 \\
\hline Não responderam & 281 & 26,63 \\
\hline Total & 1055 & 100,00 \\
\hline
\end{tabular}

\begin{tabular}{|c|c|c|}
\hline $\begin{array}{l}\text { Quando foi a última vez que você } \\
\text { ou algum outro membro de seu } \\
\text { domicílio precisou de assistência } \\
\text { ocular e não foi possível obtê-la? }\end{array}$ & Frequência & $\%$ \\
\hline$<1$ mês & 16 & 1,52 \\
\hline $1-6$ meses & 37 & 3,51 \\
\hline 6 meses - 1 ano & 47 & 4,45 \\
\hline $1-2$ anos & 46 & 4,36 \\
\hline$>2$ anos & 53 & 5,02 \\
\hline Precisou e obteve & 712 & 67,49 \\
\hline Nunca precisou & 135 & 12,80 \\
\hline Não sabe & 9 & 0,85 \\
\hline Total & 1055 & 100,00 \\
\hline
\end{tabular}

\section{Barreiras para obtenção de assistência ocular}

Quando na questão foi declarado que houve necessidade de assistência ocular e não foi possível obtê-la, foram feitas várias questões sobre os motivos que melhor explicam porque não foi possível obter assistência ocular e as principais barreiras.

A tabela 3 mostra que as principais barreiras relatadas pelos respondentes foram: não poder arcar com o custo da consulta, tentativa frustrada de obtenção de assistência ocular e dificuldade em chegar ao prestador do serviço.

\section{Local de realização da assistência ocular}

A distribuição do local de realização da assistência ocular mostra que das 273 pessoas que utilizaram serviços de assistência ocular no último ano, $90(32,97 \%)$ foram atendidas em hospitais/clínicas públicas, 69 (25,27\%) em serviços particulares sem qualquer tipo de reembolso, $62(22,71 \%)$ pelo sistema de convênio/seguro médico, $50(18,32 \%)$ em outras instituições (sindicatos ou ópticas) e $2(0,73 \%)$ não souberam informar. Dentre os $50(18,32 \%)$ respondentes que foram atendidos em outras instituições, $28(10,26 \%)$ foram atendidos em ópticas, $6(2,20 \%)$ em sindicatos e $16(5,86 \%)$ não sabiam informar.

As habilidades do prestador de assistência ocular foram consideradas adequadas por $247(90,48 \%)$ das 273 pessoas que utilizaram serviços de assistência ocular no último ano. O material médico e os equipamentos foram considerados adequados por $254(93,04 \%)$ dos entrevistados. O tempo gasto para chegar ao prestador da assistência ocular foi de 30 minutos ou menos para $250(91,59 \%)$ pessoas. Quanto ao transporte utilizado $130(47,62 \%)$ usaram transporte público, $78(28,57 \%)$ foram ao prestador andando e $65(23,81 \%)$ utilizaram algum veículo particular.

\section{Análise dos domínios de responsividade}

A frequência de avaliações positivas, levando em conta todos os domínios juntos foi de $63,37 \%$. A tabela 4 mostra a distribuição das respostas, dos 273 respondentes que utilizaram serviços de assistência ocular no último ano, para todos os domínios contidos no QRSAO. Em relação ao domínio prontidão no atendimento, $185(67,76 \%)$ acharam o tempo de viagem até o prestador do serviço de assistência ocular ótimo ou bom e $184(67,40 \%)$ o tempo de espera para ser atendido ótimo ou bom. Quase todos, 257 (94,14\%), consideraram ótimo ou bom a dignidade com que foram atendidos no serviço de assistência ocular.

Do total, $257(94,14 \%)$ consideraram ótimo ou bom o respeito a sua privacidade durante o atendimento e quanto à questão sobre poder falar de forma reservada com o prestador da assistência $225(82,72 \%)$ consideraram ótimo ou bom. Em relação ao domínio comunicação, a tabela mostra que 229 $(83,88 \%)$ consideraram ótimo ou bom a clareza com que o prestador explicou sobre o tratamento ao respondente, quanto à questão referente a ter tempo suficiente para fazer per- 
Tabela 3. Distribuição das principais barreiras para obtenção de assistência ocular

Barreiras para assistência ocular

Não pude arcar com o custo da consulta

Você tentou, mas Ihe foi negada a assistência ocular

Sem transporte / Não pude arcar com o custo do transporte

Achou que o problema na visão não era sério o suficiente para procurar tratamento

Não sabia aonde ir

Não pude sair do trabalho ou tinha outros compromissos

Outras razões

Ficou com medo de receber o tratamento

Achou que o problema na visão não poderia ser tratado

As habilidades, medicamentos ou equipamentos do prestador de assistência ocular não foram adequados

Você foi previamente mal tratado

\begin{tabular}{rc} 
Frequência & $\%$ \\
\hline 154 & 77,29 \\
84 & 42,21 \\
60 & 30,15 \\
37 & 18,59 \\
35 & 17,59 \\
33 & 16,58 \\
17 & 8,54 \\
10 & 5,03 \\
10 & 5,03 \\
7 & 3,52 \\
5 & 2,51 \\
\hline
\end{tabular}

\begin{tabular}{|c|c|c|c|c|c|c|c|}
\hline Domínios & Questão & $\begin{array}{l}\text { Ótimo } \\
\mathrm{N}(\%)\end{array}$ & $\begin{array}{l}\text { Bom } \\
N(\%)\end{array}$ & $\begin{array}{c}\text { Regular } \\
\text { N(\%) }\end{array}$ & $\begin{array}{l}\text { Ruim } \\
\mathrm{N}(\%)\end{array}$ & $\begin{array}{c}\text { Péssimo } \\
\mathrm{N}(\%)\end{array}$ & $\begin{array}{c}\text { Não sabe } \\
N(\%)\end{array}$ \\
\hline Prontidão no atendimento & $\begin{array}{l}\text { Q7312 } \\
\text { Q7313 }\end{array}$ & $\begin{array}{ll}64 & (23,44) \\
67 & (24,54)\end{array}$ & $\begin{array}{l}121(44,32) \\
117(42,86)\end{array}$ & $\begin{array}{l}46(16,85) \\
56(20,51)\end{array}$ & $\begin{array}{l}26(9,53) \\
17(6,23)\end{array}$ & $\begin{array}{ll}13 & (4,76) \\
16 & (5,86)\end{array}$ & $\begin{array}{l}3(1,10) \\
0(0,00)\end{array}$ \\
\hline Dignidade & Q7314 & $133(48,72)$ & $124(45,42)$ & $14(5,13)$ & $2(0,73)$ & $0(0,00)$ & $0(0,00)$ \\
\hline Privacidade & $\begin{array}{l}\text { Q7315 } \\
\text { Q7320 }\end{array}$ & $\begin{array}{r}119(43,59) \\
96(35,16)\end{array}$ & $\begin{array}{l}138(50,55) \\
129(47,25)\end{array}$ & $\begin{array}{l}10(3,66) \\
30(10,99)\end{array}$ & $\begin{array}{l}1(0,37) \\
9(3,30)\end{array}$ & $\begin{array}{ll}4 & (1,46) \\
8 & (2,93)\end{array}$ & $\begin{array}{ll}1 & (0,37) \\
1 & (0,37)\end{array}$ \\
\hline Comunicação & $\begin{array}{l}\text { Q7416 } \\
\text { Q7317 } \\
\text { Q7318 }\end{array}$ & $\begin{array}{r}124(45,42) \\
98(35,90) \\
68(24,91)\end{array}$ & $\begin{array}{r}105(38,46) \\
115(42,12) \\
99(36,26)\end{array}$ & $\begin{array}{ll}35 & (12,82) \\
38 & (13,92) \\
73 & (26,74)\end{array}$ & $\begin{aligned} 5 & (1,83) \\
15 & (5,49) \\
23 & (8,42)\end{aligned}$ & $\begin{array}{l}4(1,47) \\
6(2,20) \\
8(2,94)\end{array}$ & $\begin{array}{ll}0 & (0,00) \\
1 & (0,37) \\
2 & (0,73)\end{array}$ \\
\hline Autonomia & Q7319 & $68(24,91)$ & $117(42,86)$ & $63(23,07)$ & $13(4,76)$ & $10(3,66)$ & $2(0,73)$ \\
\hline Escolha & Q7321 & $78(28,57)$ & $106(38,82)$ & $59(21,62)$ & $23(8,42)$ & $5(1,84)$ & $2(0,73)$ \\
\hline Infraestrutura & $\begin{array}{l}\text { Q7322 } \\
\text { Q7323 }\end{array}$ & $\begin{array}{l}149(54,58) \\
110(40,29)\end{array}$ & $\begin{array}{l}106(38,82) \\
117(42,86)\end{array}$ & $\begin{array}{l}14(5,13) \\
29(10,62)\end{array}$ & $\begin{array}{r}1(0,37) \\
10(3,66)\end{array}$ & $\begin{array}{ll}2 & (0,73) \\
6 & (2,20)\end{array}$ & $\begin{array}{ll}1 & (0,37) \\
1 & (0,37)\end{array}$ \\
\hline
\end{tabular}

guntas sobre o problema ocular e tratamento, $213(78,02 \%)$ a consideraram ótimo ou bom e na última questão desse domínio, apenas $61,17 \%$ consideraram ótimo ou bom a experiência do respondente em conseguir informações sobre outros tipos de tratamento ou exames. No domínio autonomia, 185 $(67,77 \%)$ consideraram ótima ou boa a experiência de estar envolvido em tomar decisões sobre sua assistência ocular ou tratamento e no domínio escolha 184 (67,40\%) dos respondentes consideraram ótimo ou bom a liberdade que teve para escolher o seu prestador de assistência ocular. Em relação ao domínio infra-estrutura, 255 (93,41\%) dos respondentes classificaram como ótima ou boa a limpeza das salas dentro do local de exame, incluindo o banheiro e quanto ao espaço na sala de espera e na sala onde foram examinados $227(83,15 \%)$ consideraram ótimo ou bom.

Cem pessoas se mostraram insatisfeitas com serviços recebidos, destas $45(45,00 \%)$ procuraram e foram atendidas em hospitais/clínicas públicas, 18 (18,00\%) em hospitais/clínicas particulares, $17(17,00 \%)$ através de convênio, 19 (19,00\%) em outros tipos de estabelecimentos e 1 (1,00\%) respondente não sabia onde foi realizado o atendimento. Das 19 pessoas que relataram ter sido atendidas em outros tipos de estabelecimentos, $17(89,47 \%)$ foram atendidas em ópticas e $2(10,53 \%)$ foram em um sindicato. Quanto à distribuição de escolaridade dos indivíduos insatisfeitos: $56(56,00 \%)$ tinham até primário completo, $27(27,00 \%)$ tinham ensino médio completo, 14 $(14,00 \%)$ tinham ensino fundamental completo e $3(3,00 \%)$ tinham ensino superior completo.

\section{DISCUSSÃO}

A necessidade de assistência ocular em três distritos de baixa renda da zona leste da cidade de São Paulo foi de aproximadamente $86,00 \%$ da amostra estudada. Este número reflete a importância da visão e das doenças oculares no contexto da saúde geral.

Quanto à obtenção de assistência ocular, pode-se concluir que grande parte $78,16 \%$ dos que necessitaram de assistência ocular conseguiram obtê-la.

O principal motivo para necessidade de assistência ocular relatado por dois terços da amostra estudada foi presença de visão embaçada, reduzida ou borrada. Este achado corrobora o de outros estudos na Índia ${ }^{(9)}$ e na Austrália ${ }^{(10)}$ e estão de acordo com a classificação de visão autorrelatada em recente estudo realizado em idosos residentes na mesma área geográfica da população estudada ${ }^{(11)}$. 
As barreiras mais frequentemente apontadas pelos respondentes que necessitaram e não obtiveram assistência ocular foram o custo da consulta e a dificuldade de acesso ao serviço. Estes achados confirmam o de estudo brasileiro anterior sobre barreiras para cirurgia de catarata senil ${ }^{(12)}$ e de estudos recentes em grupos minoritários de países desenvolvi$\operatorname{dos}^{(13)}$ e de população idosa afro-americana ${ }^{(14)}$. Quando o respondente afirmou que tentou, mas não foi possível obter a assistência ocular, não foi identificado o motivo do por que foi negado o atendimento. Estes motivos devem ser investigados em futuros estudos com o aprimoramento desta ferramenta, pois esta questão pode esclarecer muitos problemas de acesso.

A dificuldade de acesso é evidenciada pela pouca oferta de serviços públicos de assistência ocular na região de estudo, onde atualmente há apenas um hospital público que oferece serviços cirúrgicos e poucas unidades básicas de saúde com médico oftalmologista prestando atendimento secundário ${ }^{(15-16)}$.

Estima-se que $80,00 \%$ da população brasileira depende de assistência médica governamental ${ }^{(17)}$, porém dos que necessitaram e obtiveram assistência ocular até um ano antes da entrevista apenas $90(32,97 \%)$ foram atendidas em hospitais/clínicas públicas. Esse dado é importante porque revela que hoje na região estudada, apesar da baixa renda familiar, $47,99 \%$ das pessoas que precisaram de atendimento oftalmológico foram atendidas em serviços particulares ou convênios, isso devido à baixa oferta de serviços públicos na região.

De maneira geral, obtivemos em nossa análise, resultados positivos. O usuário de assistência ocular apresentou uma boa frequência de avaliações positivas $(63,37 \%)$ em relação ao serviço oferecido. Esses dados corroboram os dados anteriores sobre a satisfação com o atendimento oftalmológico recebido, embora a metodologia usada e os parâmetros analisados sejam diferentes dos do presente estudo ${ }^{(18-19)}$.

O domínio prontidão no atendimento teve influência negativa no nível de satisfação das pessoas entrevistadas com o QRSAO, mostrando que o tempo de espera na recepção para a consulta é muito importante para satisfação com o atendimento recebido pelo paciente e confirmam achados prévios ${ }^{(20)}$. Vários estudos comprovam a influência do tempo de espera na satisfação do paciente ${ }^{(21-25)}$. O domínio comunicação demonstrou um frequência de avaliações positivas um pouco acima do domínio prontidão no atendimento. A informação ao paciente, por parte dos funcionários em um serviço, tem um impacto significativo na percepção da qualidade de cuidados e satisfação total dos pacientes, o que corrobora com o observado por Krishel ${ }^{(26)}$.

Os domínios dignidade, infra-estrutura e privacidade tiveram maior frequência de satisfação, mostrando que as pessoas que conseguiram assistência ocular avaliaram esses pontos como positivos e de excelente qualidade em praticamente todas as instituições prestadoras de assistência, o mesmo foi encontrado por alguns autores ${ }^{(1,18)}$.

Trabalho realizado por outros autores relatou que as maiores diferenças na frequência de pessoas satisfeitas foram encontradas observando-se que o grau de satisfação é tanto menor quanto melhor o nível de instrução, porém nosso estudo mostra que as pessoas com pior nível de instrução ficaram menos satisfeitas com a assistência ocular recebida quando comparado aos outros níveis de escolaridade ${ }^{(1)}$. Boa parte das pessoas insatisfeitas foi atendida em instituições públicas $(45,00 \%)$. A escolaridade foi de até primário completo em $56,00 \%$ das pessoas insatisfeitas mostrando que o nível cultural pode influenciar a satisfação das pessoas com o serviço recebido ${ }^{(20)}$.

O QRSAO foi desenvolvido pela OMS a partir do questionário da Pesquisa Mundial de Saúde ${ }^{(1)}$ e foi aplicado pela primeira vez no Brasil para avaliação do sistema de assistência ocular neste estudo, mostrando-se um instrumento valioso e importante na busca da qualidade no atendimento ao paciente.

O uso de questionários, atualmente, já está bastante difundido na área da saúde, porque são instrumentos que servem para conhecer e satisfazer as necessidades dos pacientes, como estratégias de ação para se atingir a qualidade de serviços percebida pelos pacientes, contribuindo para a diminuição da insatisfação e incredulidade dos pacientes diante dos serviços oferecidos ${ }^{(27-30)}$.

\section{CONCLUSÃO}

A assistência oftalmológica é um serviço especializado bastante procurado pelas pessoas residentes na região estudada e aproximadamente dois terços das pessoas que necessitaram e obtiveram essa assistência nos últimos 12 meses mostraram-se satisfeitos com o serviço prestado.

As principais barreiras para obtenção da assistência ocular relatadas foram o custo da consulta e falta de acesso aos serviços.

Alguns pontos foram bastante positivos na avaliação dos serviços de assistência ocular da região estudada. O tempo gasto no transporte pelo paciente para chegar ao prestador da assistência ocular foi curto; os domínios dignidade, infraestrutura e privacidade tiveram uma frequência de satisfação elevada e o principal motivo de insatisfação foi a demora para o atendimento.

\section{AGRADECIMENTOS}

Os autores agradecem a Matheus Machado Oliveira, Sávia Maria Emrich, Lucas de Toledo Lima, Guilherme Oliveira, Marcos Milazzo e Ana Carolina Manzoli pela colaboração nos procedimentos de enumeração, visitas e entrevistas domiciliares.

\section{ABSTRACT}

Purpose: To evaluate eye care services from the user's perspective in a low income population from the east zone of the city of São Paulo - Brazil. Methods: A household survey was 
performed using cluster sampling in three low income districts of the city of São Paulo - Brazil. From July/2004 to January/ 2005, 1055 interviews with an adult household representative were carried and an eye care system responsiveness questionnaire was administered through individual interview. Results: $71.56 \%$ of the participants were women. Respondents' age ranged from 18 to 92 years (41.42 \pm 15.67 years). Regarding schooling, 525 (49.77\%) had four years or less; 489 (46.35\%) between five and eleven years, 40 (3.79\%) had eleven or more years of study. Eye care services need was reported as $712(67.49 \%)$ declaring themselves or someone else of the household needing and obtaining services and 135 (12.80\%) had never needed eye care. The most frequently cited barriers to obtain the eye care service by respondents was cost (77.29\%), followed by unsuccessful attempt to obtain eye care (42.21\%). General satisfaction for the criteria contained in the questionnaire was $63.37 \%$. In the $36.63 \%$ dissatisfied respondents, the most frequently cited claim was the amount of time waited before consultation. Conclusion: The main barriers to obtain eye care services were cost of medical appointment and lack of access to the services. $63.37 \%$ of the individuals in need who had received eye care in last 12 months were satisfied with the service provided.

Keywords: Patient satisfaction; Eye health services; Quality of health care; Quality assurance, health care; Eye diseases/ epidemiology; Urban health services

\section{REFERÊNCIAS}

1. Szwarcwald CL, Viacava F, Vasconcellos MTL, Leal MC, Azevedo LO, Queiroz RS, et al. Pesquisa Mundial de Saúde 2003. O Brasil em números. Radis [Internet]. 2004;23, Jul. [citado 2008 Nov 23]. Disponível em: http:// www4.ensp.fiocruz.br/radis/23-capa.html.

2. Valentine NB, de Silva A, Kawabata K, Darby C, Murray CJ, Evans DB. Health system responsiveness: concepts, domains and measurement. In: Murray CJ, Evans DB, editors. Health systems performance assessment: debates, methods and empiricism. Geneva: World Health Organization, 2003.

3. World Health Organization. Background Paper for the Technical Consultation on Responsiveness Concepts and Measurement [Internet]. Geneva, Switzerland, 13-14 Sep. 2001. [cited 2006 May 5]. Available from: http://www.who.int/healthsystems-performance/technical_consultations/responsiveness_background.pdf

4. Temporini ER, Kara-José N. A perda da visão: estratégias de prevenção. Arq Bras Oftalmol. 2004;67(4):597-601.

5. Lemme AC, Noronha G, Resende JB. A satisfação do usuário em Hospital Universitário. Rev Saúde Pública. 1991;25(1):41-6.

6. Akerman M, Nadanovsky P. Avaliação dos serviços de saúde: avaliar o quê? Cad Saúde Pública. 1992;8(4):361-5.

7. Ramos DD, Lima MA. Acesso e acolhimento aos usuários em uma unidade de saúde de Porto Alegre, Rio Grande do Sul, Brasil. Cad Saúde Pública. 2003;19(1):27-34.

8. Tay T, Rochtchina E, Mitchell P, Lindley R, Wang JJ. Eye care service utilization in older people seeking aged care. Clin Experiment Ophthalmol. 2006;34(2):141-5.
9. Dandona R, Dandona L, Naduvilath TJ, McCarty CA, Rao GN. Utilization of eye care services in an urban population in southern India: the Andhra Pradesh eye disease study. Br J Ophthalmol. 2000;84(1):22-7.

10. Keeffe JE, Weih LM, McCarty CA, Taylor HR. Utilization of eye care services by urban and rural Australians. Br J Ophthalmol. 2002;86(1):24-7.

11. Cinoto RW, Berezovsky A, Belfort Jr R, Salomão SR. Comparação entre qualidade de visão auto-relatada e acuidade visual em população idosa de baixa renda na cidade de São Paulo. Arq Bras Oftalmol. 2006;69(1):17-22.

12. Lima DMG, Ventura LO, Brandt CT. Barreiras para o acesso ao tratamento da catarata senil na Fundação Altino Ventura. Arq Bras Oftalmol. 2005;68(3): 357-62.

13. Patel I, Turano KA, Broman AT, Bandeen-Roche K, Muñoz B, West SK. Measures of visual function and percentage of preferred walking speed in older adults: the Salisbury Eye Evaluation Project. Invest Ophthalmol Vis Sci. 2006; 47(1):65-71.

14. Owsley C, McGwin G, Scilley K, Girkin CA, Phillips JM, Searcey K. Perceived barriers to care and attitudes about vision and eye care: focus groups with older African Americans and eye care providers. Invest Ophthalmol Vis Sci. 2006;47(7):2797-802.

15. Rizzo, RGM. Estratégia Administrativa para Atendimento Oftalmológico do paciente usuário do Sistema Único de Saúde (SUS) nos bairros: Guaianases, São Mateus, Itaquera, Cidade Líder e Cidade Tiradentes [tese]. São Paulo: Universidade Federal de São Paulo, Escola Paulista de Medicina. 2004.

16. Petroni, FGM. Estratégia administrativa para atendimento oftalmológico do paciente usuário do Sistema Único de Saúde (SUS) nos bairros: Moóca, Penha, Vila Formosa e Vila Matilde [tese]. São Paulo: Universidade Federal de São Paulo - Escola Paulista de Medicina; 2003.

17. Kara-José N, Temporini ER. Catarata e cegueira: epidemiologia e prevenção. In: Arieta CEL. Cristalino e catarata. Rio de Janeiro: Cultura Médica. 2002; p.50-6.

18. Milhomem SC. Avaliação da qualidade de atendimento em setor de oftalmologia baseada em informação colhida por questionário dirigido [tese]. São Paulo: Universidade Federal de São Paulo - Escola Paulista de Medicina; 2003.

19. Silva LMP, Muccioli C, Belfort Jr R. Perfil socioeconômico e satisfação dos pacientes atendidos no mutirão de catarata do Instituto da Visão - UNIFESP. Arq Bras Oftalmol. 2004;67(5):737-44.

20. Martinez Ramirez A, Van-Dick Puga MA, Nápoles Rodrigues F, Robles Uribes J, Ramos Ramos A, Villaseñor Urrea I. Hacia una estrategia de garantía de calidad: satisfacción en la utilización de los servicios médicos. Cad Saúde Pública. 1996;12(3):399-403.

21. Vuori H. Patient satisfaction-an attribute or indicator of the quality of care? QRB Qual Rev Bull. 1987;13(3):106-8.

22. Yamamoto E. Os novos médicos administradores: cuidando da saúde dos pacientes e das empresas. São Paulo: Futura; 2001.

23. Young PC, Wasserman RC, McAullife T, Long J, Hagan JF, Health B. Why families change pediatricians. Factors causing dissatisfaction with pediatric care. Am J Dis Child. 1985;139(7):683-6.

24. McMillian JR, Younger MS, DeWine LC. Satisfaction with hospital emergency department as a function of patient triage. Health Care Manage Rev. 1986; 11(3):21-7.

25. Piper LE. Waiting time in out patient care: a study of divergent perspectives. Mil Med. 1989;154(8):401-3.

26. Krishel S, Baraff LJ. Effect of emergency department information on patient satisfaction. Ann Emerg Med. 1993;22(3):568-72.

27. Bursch B, Beezy J, Shaw R. Emergency department satisfaction: what matters most? Ann Emerg Med. 1993;22(3):586-91.

28. Kroenke K, Stump T, Clark DO, Callahan CM, McDonald CJ. Symptoms in hospitalized patients: outcome and satisfaction with care. Am J Med. 1999; 107(5):425-31. Comment in: Am J Med. 1999;107(5):519-21.

29. Boudreaux ED, Ary RD, Mandry CV, McCabe B. Determinants of patient satisfaction in a large municipal ED: the role of demographic variables, visit characteristics, and patient perceptions. Am J Emerg Med. 2000;18(4):394-400.

30. Counselman FL, Graffeo CA, Hill JT. Patient satisfaction with physician assistants (PAs) in an ED fast track. Am J Emerg Med. 2000;18(4):661-5. 